\title{
Cell-free mixing of Escherichia coli crude extracts to prototype and rationally engineer high-titer mevalonate synthesis
}

\author{
Quentin M. Dudley ${ }^{1}$, Kim C. Anderson ${ }^{1}$, Michael C. Jewett ${ }^{1,2,3,4^{*}}$
}

${ }^{1}$ Department of Chemical and Biological Engineering, ${ }^{2}$ Chemistry of Life Processes Institute, Northwestern University, Evanston, IL 60208, USA, ${ }^{3}$ Robert H. Lurie Comprehensive Cancer Center, ${ }^{4}$ Simpson Querrey Institute Northwestern University, Chicago, IL 60611, USA

${ }^{*}$ Correspondence: Michael C. Jewett, Department of Chemical and Biological Engineering, Northwestern University, Evanston, IL 60208, USA

E-mail addresses: m-jewett@northwestern.edu

Tel: 1847467 5007; Fax: 18474913728

\section{Supplementary Information}

\author{
Supplementary Figures S1-7 \\ Supplementary Table $\mathbf{S 1}$ \\ Appendices S1-3
}




\section{Supplementary Figures}

\section{Figure S1.}

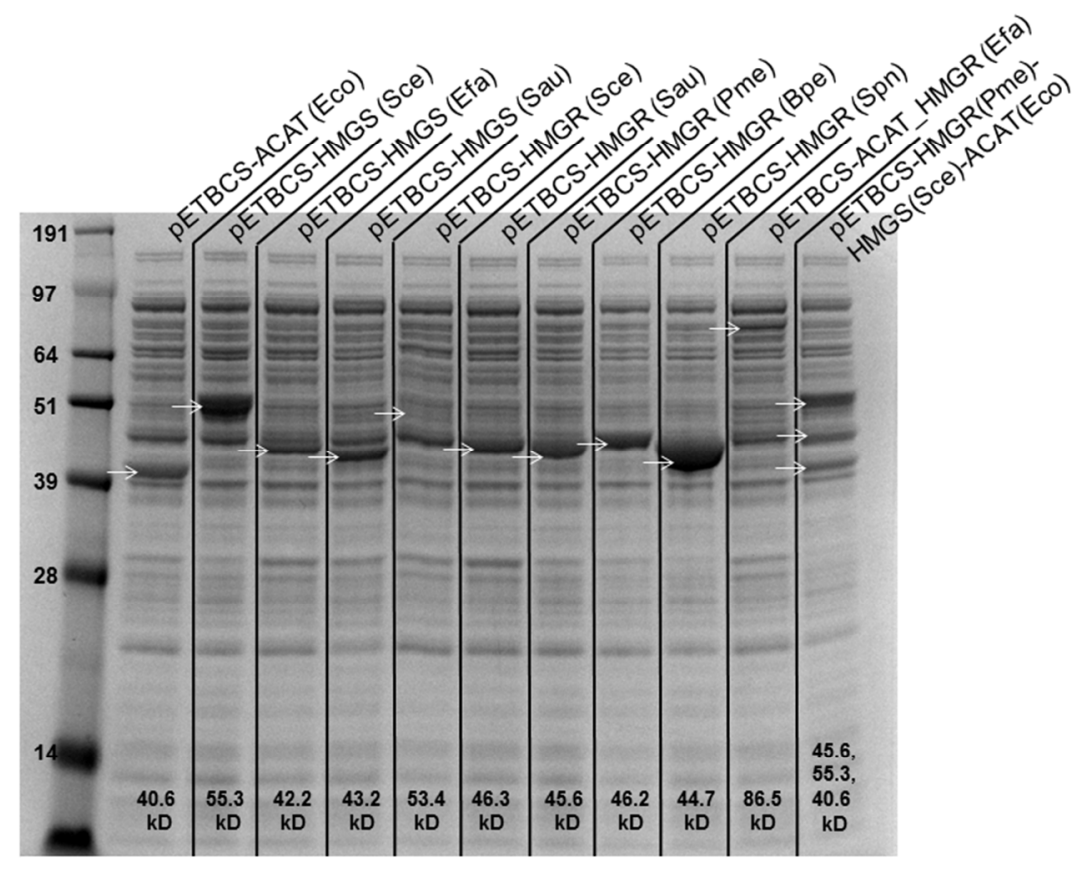

Figure S1. SDS-PAGE gel of crude lysates used in this study. Lysates were generated as described in the Materials and Methods section. In brief, E. coli containing the appropriate pETBCS-based plasmid were grown in 1-liter shake flasks at $37^{\circ} \mathrm{C}$ in rich media containing $100 \mu \mathrm{g} \cdot \mathrm{L}^{-1}$ carbenicillin. Cultures were induced with $0.1 \mathrm{mM}$ IPTG at $0.6 \mathrm{OD}_{600 \mathrm{~nm}}$ and growth continued at $30{ }^{\circ} \mathrm{C}$ until harvest. Four hours post-induction, cultures were pelleted by centrifugation ( $8000 \times g$ for 15 minutes) and the resulting pellet was rinsed twice in S30 buffer and flash-frozen. To generate crude extracts, cell pellets were thawed on ice and suspended in $\mathrm{S} 30$ buffer $(0.8 \mathrm{~mL}$ per gram cell pellet), and lysed at 20,000 psi (homogenizing pressure) using an EmulsiFlex-B15 homogenizer. Total protein was quantified by Bradford assay with bovine serum albumin (BSA) as the standard using a microplate protocol (Bio-Rad, Hercules, CA). The image depicted is a NuPAGE $®$ Novex $12 \%$ Bis-Tris SDS-PAGE protein gel run in MOPS buffer and stained with SimplyBlue SafeStain (Coomassie). Each lane was loaded with $10 \mu \mathrm{g}$ total protein from the extract (soluble protein only); arrows indicate protein overexpression at expected size. 
Figure S2.

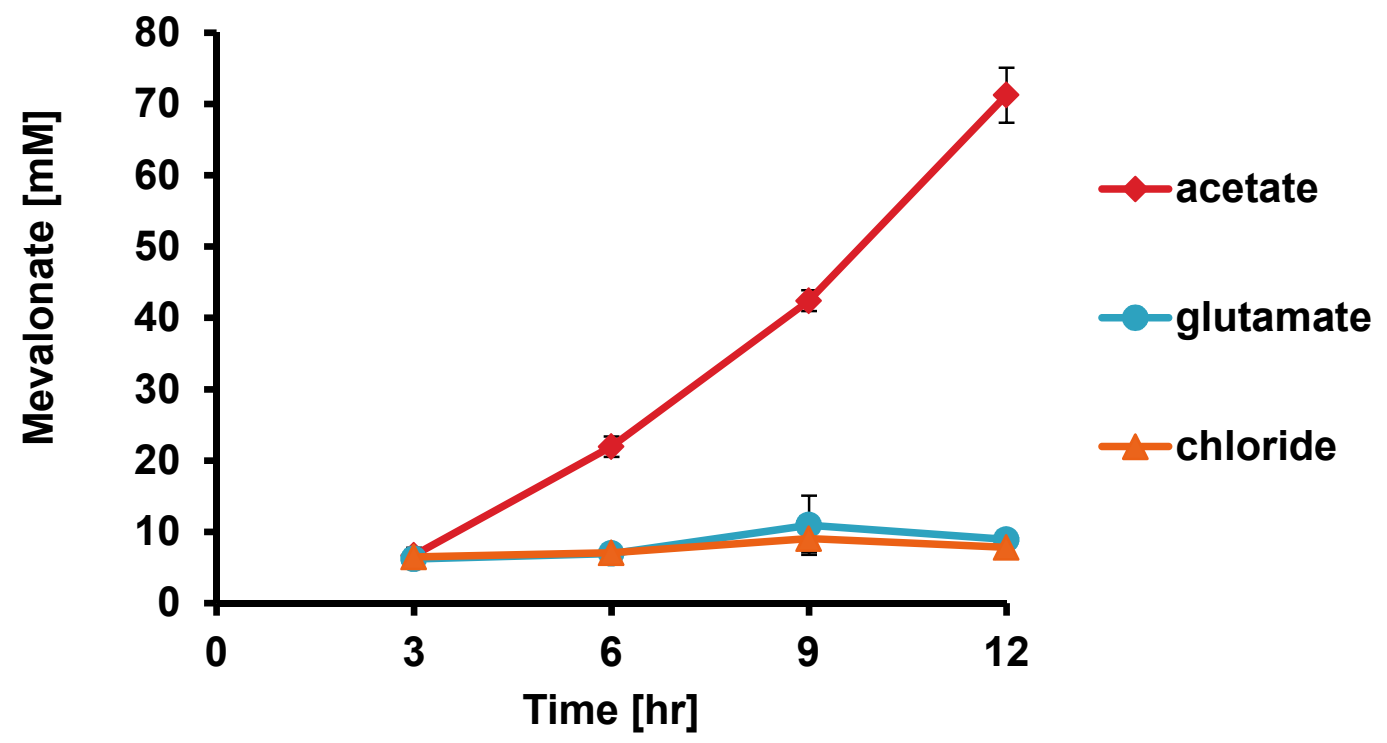

Figure S2. Evaluation of chloride, glutamate, and acetate salts in the CFME reaction. Cell free reactions were performed at a volume of $25 \mu \mathrm{L}$ in $1.5 \mathrm{~mL}$ Eppendorf tubes and incubated at $37{ }^{\circ} \mathrm{C}$. The standard reaction contained the following components: $200 \mathrm{mM}$ glucose, salts, $10 \mathrm{mM}$ potassium phosphate $\left(\mathrm{K}_{2} \mathrm{HPO}_{4}, \mathrm{pH} 7.2\right.$ (Calhoun and Swartz, 2005), 1mM NAD $+1 \mathrm{mM}$ ATP, $1 \mathrm{mM}$ CoA. Three extracts with overexpression of $\mathrm{ACAT}(\mathrm{Eco}), \mathrm{HMGS}(\mathrm{Sce}), \mathrm{HMGR}(\mathrm{Pme})$ mixed at equal ratios (10 $\mathrm{mg} / \mathrm{mL}$ total protein concentration in extract). Cation concentrations in the salts were set at $8 \mathrm{mM} \mathrm{Mg}^{2+}, 10 \mathrm{mM} \mathrm{NH}_{4}{ }^{+}$, and $134 \mathrm{mM} \mathrm{K}^{+}$(Kay and Jewett, 2015) and are consistent with principles of cytoplasmic mimicry (Jewett et al., 2008; Jewett and Swartz, 2004). The counter ions (acetate, glutamate, or chloride) to the $\mathrm{Mg}^{2+}, \mathrm{NH}_{4}{ }^{+}$, and $\mathrm{K}^{+}$were varied as indicated in the figure legend. Values represent averages $(n=2)$ and error bars represent the 1 s.d. 


\section{Figure S3.}
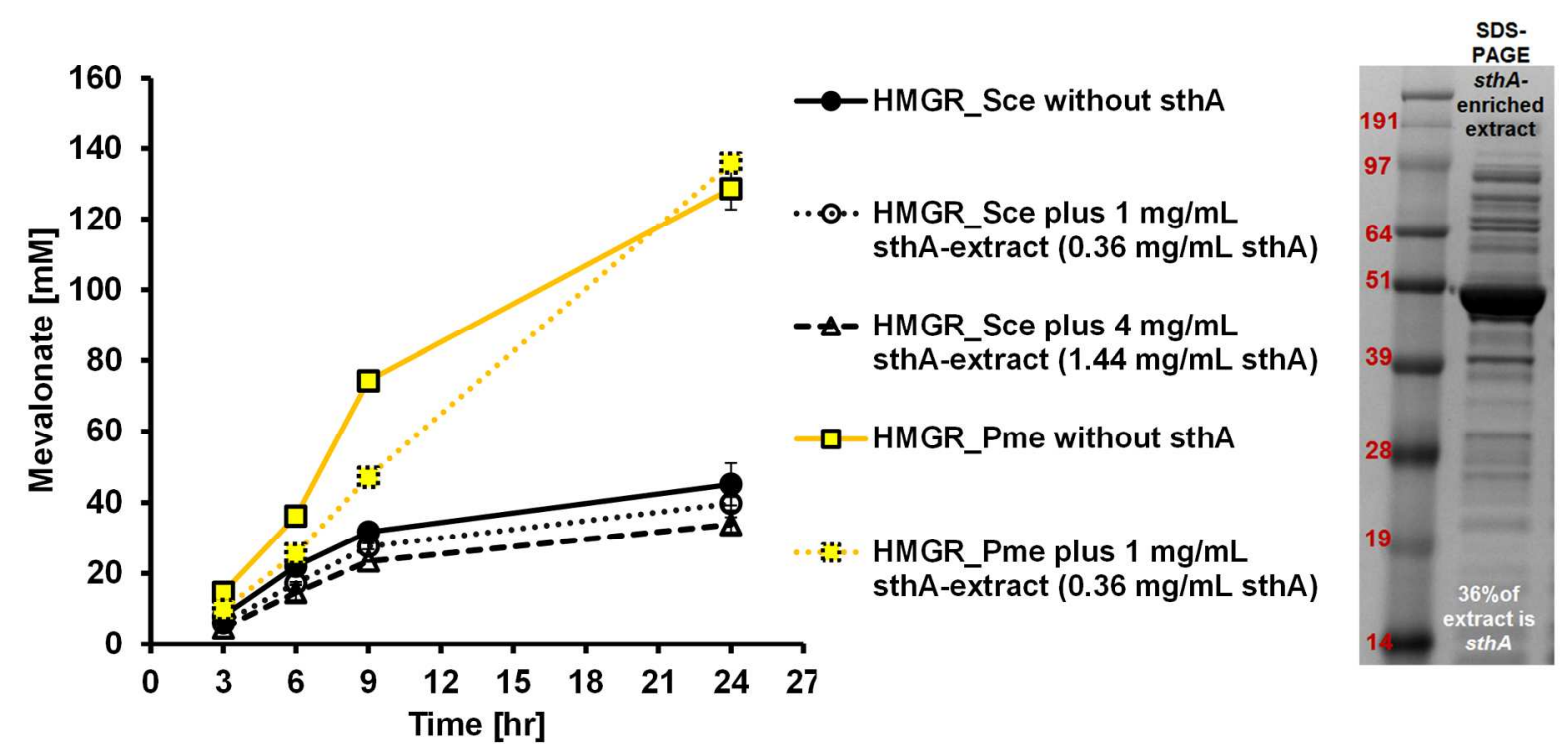

Figure S3. Addition of soluble transhydrogenase (sthA)-enriched extracts. Cell free reactions were performed using three mixed extracts as in Figure 2 of the manuscript. All reactions used ACAT from E. coli and HMGS from S. cerevisiae while the legend denotes if HMGR is from $S$. cerevisiae or $P$. mevalonii. For reactions depicted by circles and squares, each extract contributed $3.0 \mathrm{mg} \cdot \mathrm{mL}^{-1}$ of total protein to the reaction. Reactions without sthA were supplemented with $1 \mathrm{mg} \cdot \mathrm{mL}^{-1} \mathrm{BL} 21$ (DE3) extract with no expressed plasmid while reactions "plus sthA-extract" contained 1 $\mathrm{mg} \cdot \mathrm{mL}^{-1}$ extract with sthA overexpressed using the same pETBCS plasmid as other extracts reported. In the reaction represented by triangles, the ACAT, HMGS, and HMGR extracts each contribute $2.0 \mathrm{mg} \cdot \mathrm{mL}^{-1}$ of total protein and are combined with 4.0 $\mathrm{mg} \cdot \mathrm{mL}^{-1}$ sthA-enriched lysate. The insert SDS-PAGE gel shows overexpression of $\operatorname{sth} A$ in the lysate. Reactions also contained the standard salts, cofactors, and buffers: 200 $\mathrm{mM}$ glucose, acetate salts $(8 \mathrm{mM}$ magnesium acetate, $10 \mathrm{mM}$ ammonium acetate, 134 $\mathrm{mM}$ potassium acetate), $10 \mathrm{mM}$ potassium phosphate $\left(\mathrm{K}_{2} \mathrm{HPO}_{4}, \mathrm{pH} 7.2\right), 1 \mathrm{mM} \mathrm{NAD}{ }^{+}$, $1 \mathrm{mM}$ ATP, $1 \mathrm{mM}$ CoA, and $100 \mathrm{mM}$ Bis-Tris buffer. Values represent averages $(n=3)$ and error bars represent 1 s.d. 


\section{Figure S4.}

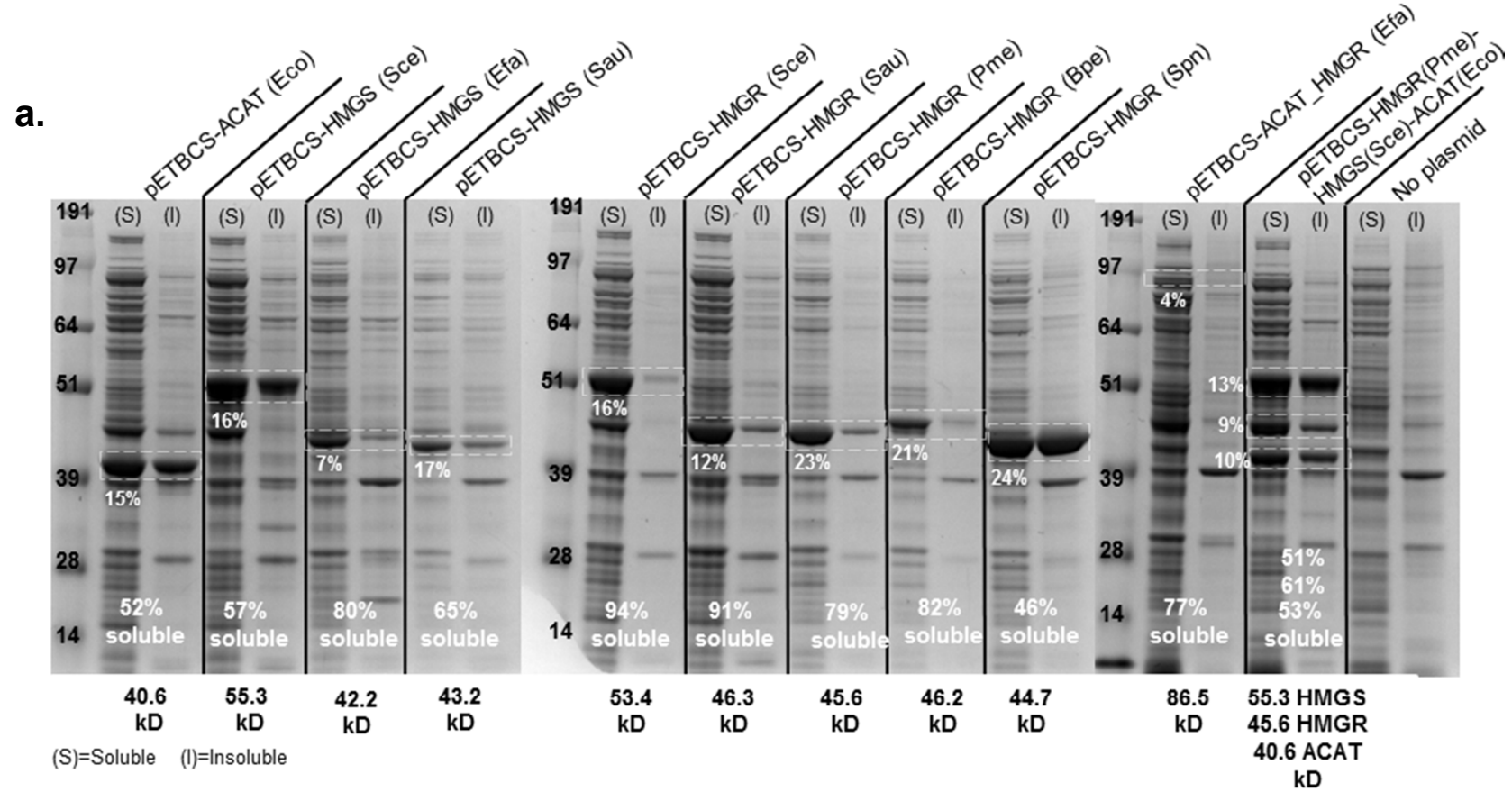

Figure S4. SDS-PAGE gel of soluble (S) and insoluble (I) protein in cells upon lysis. E. coli containing the appropriate pETBCS-based plasmid were grown in $5 \mathrm{~mL}$ culture tubes at $37{ }^{\circ} \mathrm{C}$ in rich media containing $100 \mu \mathrm{g} \cdot \mathrm{L}^{-1}$ carbenicillin. Cultures were induced with $0.1 \mathrm{mM}$ IPTG at 0.6

b.

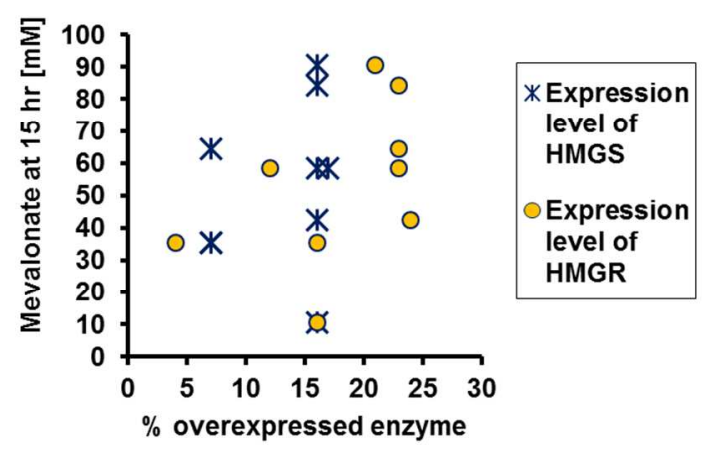
$\mathrm{OD}_{600 \mathrm{~nm}}$ and growth continued at $30{ }^{\circ} \mathrm{C}$ until harvest. Four hours post-induction, cultures were pelleted by centrifugation ( $4000 \times g$ for 10 minutes) and the resulting pellet was rinsed in PBS buffer. Pellets were resuspended in $250 \mu \mathrm{L}$ PBS and sonicated to lyse cells. The mixture was centrifuged at $21000 \times g$ for 10 minutes, the supernatant constitutes the soluble fraction. The remaining pellet was rinsed twice in buffer and resuspended in $250 \mu \mathrm{L}$ PBS with $2 \%$ SDS and heated to $90{ }^{\circ} \mathrm{C}$ for 10 minutes to solubilize all proteins in the pellet. (a) The image depicted is a NuPAGE ${ }^{\circledR}$ Novex $10 \%$ Bis-Tris SDS-PAGE protein gel run in MOPS buffer and stained with SimplyBlue SafeStain (Coomassie). ImageJ software (U. S. National Institutes of Health, Bethesda, Maryland, USA, http://imagej.nih.gov/ij/) was used to measure the relative area of overexpressed protein bands (boxes are around the overexpressed protein). 
Percentages in the soluble lane indicate the relative amount of overexpressed protein relative to all proteins in the lane. At the bottom of the gel in each pair of lanes is a percentage comparing the area of soluble versus insoluble overexpressed protein. (b) Mevalonate titers are plotted as a function of overexpressed enzyme in each extract. 
Figure S5.

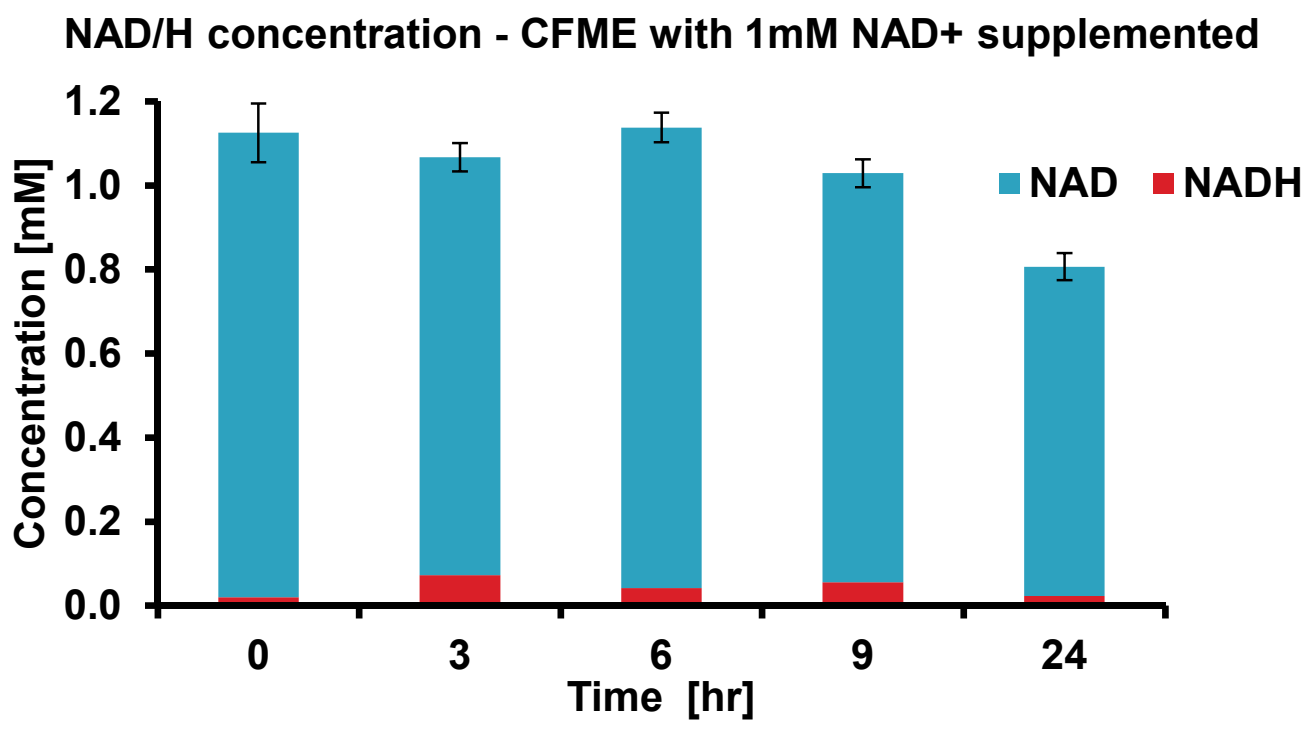

NAD/H concentration - CFME with no NAD+ supplemented

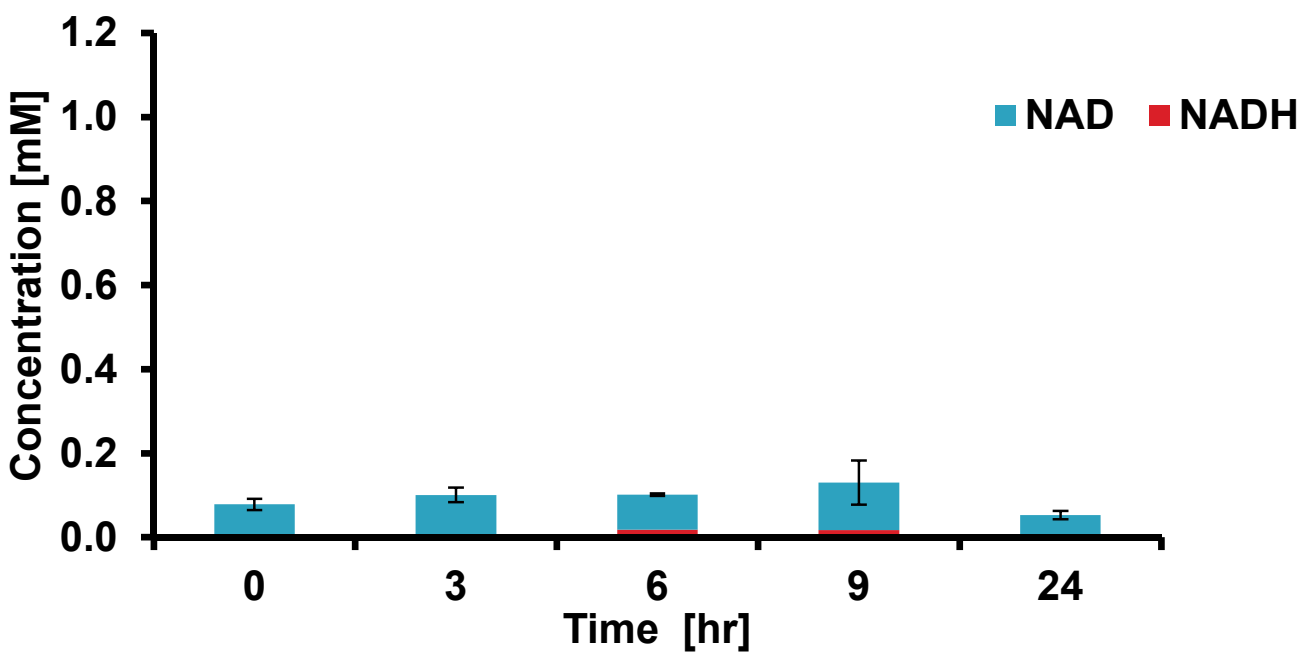

Figure S5. Concentration of $\mathrm{NAD}^{+}$and NADH throughout a typical CFME reaction. $\mathrm{NAD}^{+}$and $\mathrm{NADH}$ were quantified using a fluorescent kit (Sigma). Cell-free reactions were quenched by diluting 1:200 in the kit extraction buffer and immediately freezing in liquid nitrogen. Samples were then thawed and prepared following the kit protocol. Values represent averages $(n=3)$ and error bars represent 1 s.d. 
Figure S6.
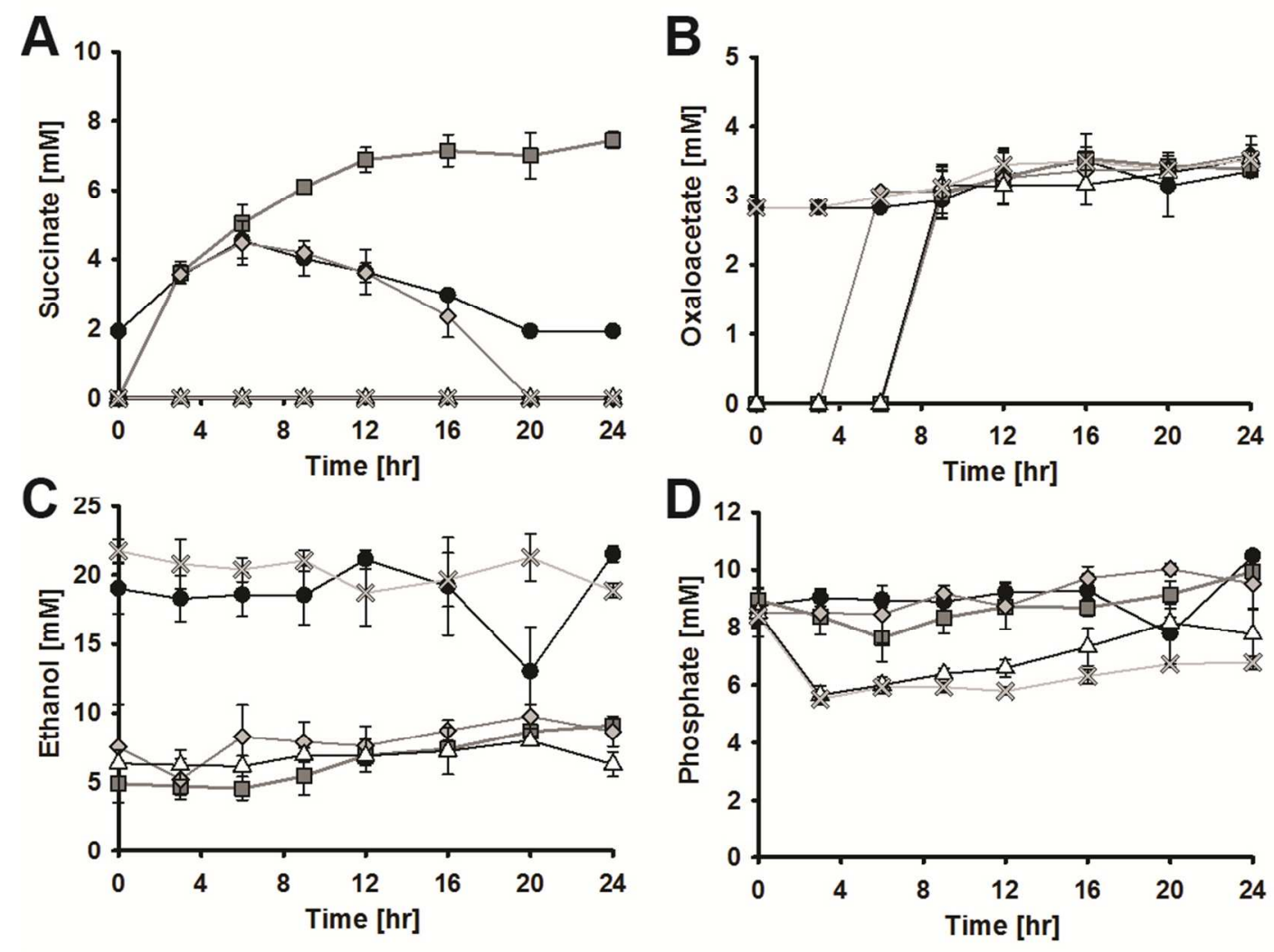

Wild Type $\rightarrow-\Delta$ IdhA $\diamond-\Delta$ acs

$-\triangle \sim \Delta$ ackA-pta

* $\Delta$ acs $\triangle$ ackA-pta

Figure S6. Concentrations of metabolites over time within a cell free reaction. (A) Succinate (B) Oxaloacetate (C) Ethanol (D) Phosphate. Source strain genome engineering can redirect metabolic flux in the cell free reaction. Knockout of $I d h A$ eliminates lactate accumulation and causes a small amount of succinate to be generated. Knockout of ackA-pta eliminates acetate consumption. Acetate consumption is hypothesized to be a necessary sink for excess ATP generated by the glycolysis phase of the pathway. Values represent averages $(n=3)$ and error bars represent 1 s.d. 
Figure S7.

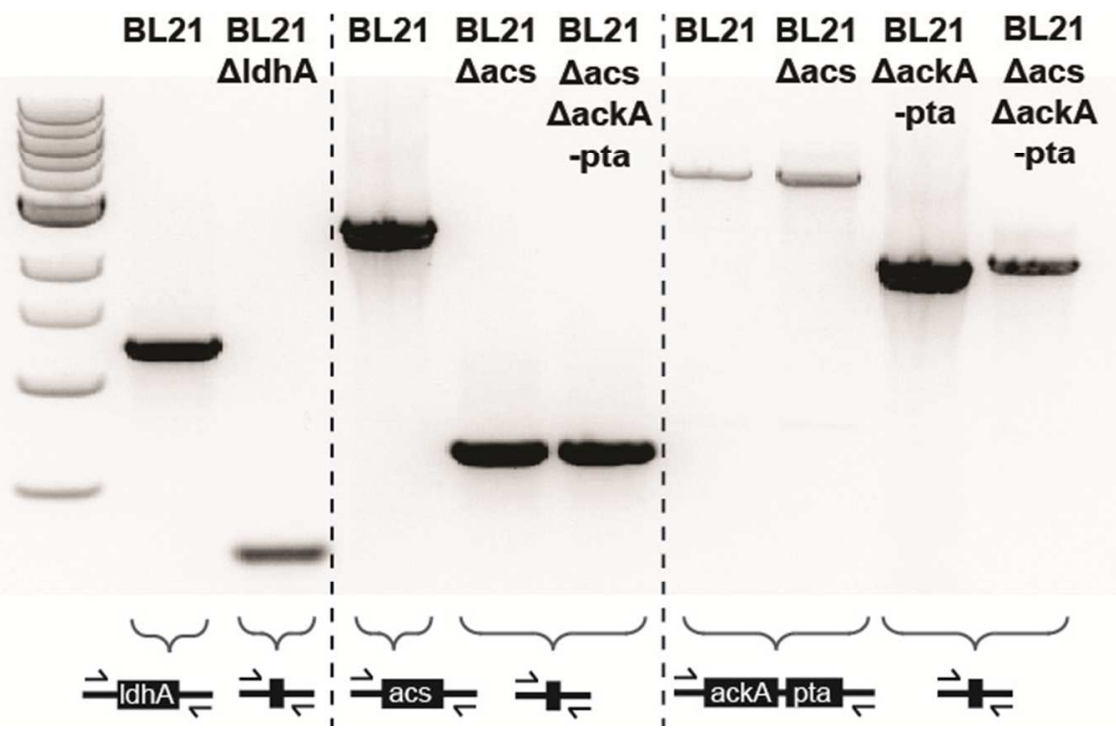

Figure S7. PCR amplification of genomic regions where target genes were replaced by a scar sequence (containing the FRT sequence) using the DatsenkoWanner technique (Datsenko and Wanner, 2000). In addition to correct size of PCR amplicons, genomic regions were sequenced (Appendix S2). 


\section{Supplementary Tables}

Table S1. Selected titers and relative productivities from literature

\begin{tabular}{|c|c|c|c|c|}
\hline $\begin{array}{l}\text { Final } \\
\text { Titer } \\
\text { (g/L) }\end{array}$ & Product / Organism & $\begin{array}{c}\text { Time } \\
\text { (hr) }\end{array}$ & $\begin{array}{c}\text { Relative } \\
\text { Productivity } \\
\text { (mg/L/hr) }^{*}\end{array}$ & Reference \\
\hline 0.1 & amorphadiene / E. coli & 12 & 20 & (Martin et al., 2003) \\
\hline 0.7 & mevalonate / E. coli ** & 80 & 9 & (Dueber et al., 2009) \\
\hline 45 & amorphadiene / yeast & 110 & 891 & (Westfall et al., 2012) \\
\hline 0.6 & amorphadiene / E. coli & 72 & 18 & (Woo et al., 2013) \\
\hline 25 & artemisinic acid / yeast & 140 & 339 & (Paddon et al., 2013) \\
\hline 47 & mevalonate / E. coli & 50 & 940 & (Tabata and Hashimoto, 2004) \\
\hline 88 & mevalonate / E. coli & 48 & 1833 & (Xiong et al., 2014) \\
\hline 0.036 & mevalonate / yeast & $70 ?$ & $0.5 ?$ & (Rodriguez et al., 2016) \\
\hline 16 & mevalonate / CFME E. coli & 20 & 815 & This study \\
\hline
\end{tabular}




\section{Appendices}

\section{Appendix S1. Gene Sequences}

Note: ribosome binding site is denoted in lowercase

ACAT from Escherichia coli (Dueber et al., 2009), E.C. Number: 2.3.1.9

Salis rbs strength: $3102.82 \mathrm{au}$

atataacacacataaggaggatctATGAAGAACTGTGTGATTGTTTCTGCGGTCCGCACGGC GATCGGCAGCTTTAACGGCTCTTTAGCGAGCACCTCTGCAATCGATCTGGGTGCG ACGGTCATTAAGGCCGCCATTGAACGCGCCAAAATCGACAGCCAGCACGTTGATG AGGTGATCATGGGCAATGTGTTACAAGCCGGCCTGGGTCAAAACCCAGCGCGTCA AGCACTGTTAAAATCTGGTCTGGCCGAGACCGTGTGTGGCTTCACCGTCAATAAG GTTTGCGGCTCTGGCCTGAAGAGCGTGGCCCTGGCAGCACAAGCGATTCAAGCC GGTCAGGCACAAAGCATCGTTGCGGGTGGCATGGAGAACATGTCTCTGGCGCCG TACTTATTAGATGCCAAAGCCCGCAGCGGTTATCGCCTGGGCGATGGTCAGGTGT ACGACGTCATCTTACGCGATGGCTTAATGTGCGCGACCCACGGTTACCACATGGG TATTACGGCCGAAAACGTGGCGAAAGAATACGGCATTACGCGCGAGATGCAGGAT GAATTAGCACTGCACTCTCAGCGCAAAGCAGCAGCCGCGATCGAGTCTGGTGCGT TTACGGCGGAAATCGTGCCAGTTAACGTGGTCACGCGCAAGAAGACGTTCGTTTT CAGCCAGGACGAGTTCCCGAAGGCAAACAGCACCGCGGAGGCCTTAGGTGCCTT ACGCCCAGCCTTTGACAAAGCGGGCACGGTCACCGCCGGTAATGCGAGCGGCAT CAATGATGGTGCAGCGGCACTGGTCATCATGGAAGAGAGCGCCGCATTAGCAGC GGGTCTGACCCCATTAGCGCGCATTAAATCTTATGCCAGCGGCGGCGTCCCACCA GCCCTGATGGGCATGGGTCCGGTCCCAGCCACGCAAAAAGCCCTGCAATTAGCG GGCCTGCAACTGGCCGACATTGATCTGATCGAGGCGAACGAGGCGTTTGCAGCG CAGTTCCTGGCGGTGGGTAAGAATCTGGGCTTCGACAGCGAGAAAGTCAATGTGA ACGGTGGCGCGATTGCGTTAGGCCATCCGATTGGTGCAAGCGGCGCACGCATCTT AGTGACGTTACTGCACGCCATGCAGGCACGCGACAAGACCTTAGGCCTGGCGAC CTTATGTATTGGTGGCGGTCAAGGTATCGCCATGGTGATCGAACGCCTGAACCCTA GGTGA

HMGS from Saccharomyces cerevisiae (Dueber et al., 2009) E.C. Number: 2.3.3.10 Salis rbs strength: 29308.88 au aacggtcacaataaggagggatctATGAAACTGAGCACCAAGCTGTGCTGGTGTGGCATCAA GGGTCGCCTGCGCCCACAAAAGCAGCAACAGCTGCACAACACGAACCTGCAAATG ACCGAGCTGAAAAAGCAGAAGACGGCCGAGCAAAAGACCCGCCCGCAGAACGTT GGCATCAAGGGCATCCAGATTTATATCCCGACGCAGTGTGTCAACCAATCTGAGCT GGAGAAATTCGATGGCGTCAGCCAGGGTAAGTACACCATCGGCCTGGGCCAGAC CAACATGAGCTTCGTGAACGACCGTGAGGACATCTATTCTATGAGCCTGACGGTG CTGTCTAAGCTGATCAAGAGCTACAACATCGACACGAATAAGATCGGTCGTCTGGA GGTGGGTACGGAGACGCTGATTGACAAGAGCAAAAGCGTGAAGTCTGTCTTAATG CAGCTGTTCGGCGAGAACACGGATGTCGAGGGTATCGACACCCTGAACGCGTGTT ACGGCGGCACCAACGCACTGTTCAATAGCCTGAACTGGATTGAGAGCAACGCCTG GGATGGCCGCGATGCGATCGTCGTGTGCGGCGATATCGCCATCTATGACAAGGGT GCGGCACGTCCGACCGGCGGTGCAGGCACCGTTGCGATGTGGATTGGCCCGGAC 
GCACCAATTGTCTTCGATTCTGTCCGCGCGTCTTACATGGAGCACGCCTACGACTT TTACAAGCCGGACTTCACGAGCGAATACCCGTACGTGGACGGCCACTTCTCTCTG ACCTGCTATGTGAAGGCGCTGGACCAGGTTTATAAGTCTTATAGCAAAAAGGCGAT TTCTAAGGGCCTGGTCAGCGACCCGGCAGGCAGCGACGCCCTGAACGTGCTGAA GTATTTCGACTACAACGTGTTCCATGTCCCGACCTGCAAATTAGTGACCAAATCTTA TGGCCGCCTGTTATATAATGATTTCCGTGCCAACCCGCAGCTGTTCCCGGAGGTTG ACGCCGAGCTGGCGACGCGTGATTACGACGAGAGCCTGACCGACAAGAACATCG AGAAGACCTTCGTCAACGTCGCGAAGCCGTTCCACAAAGAGCGTGTGGCCCAAAG CCTGATCGTCCCGACCAACACGGGCAACATGTATACCGCGTCTGTCTACGCGGCA TTCGCGAGCCTGCTGAATTACGTCGGTTCTGACGACCTGCAGGGCAAGCGCGTTG GCCTGTTCAGCTACGGTAGCGGCTTAGCGGCCAGCCTGTATAGCTGCAAAATTGT CGGCGACGTCCAGCACATCATCAAGGAGCTGGACATCACCAACAAGCTGGCGAAG CGCATCACCGAGACGCCGAAAGATTACGAGGCAGCGATCGAGTTACGCGAGAATG CGCATCTGAAGAAGAACTTCAAGCCGCAAGGTAGCATCGAGCACCTGCAGAGCGG CGTCTACTACCTGACGAACATTGACGACAAGTTCCGCCGTTCTTATGACGTCAAAA AGCTGGAGTGA

HMGS (mvaS gene) from Enterococcus faecalis (Zurbriggen et al., 2012) E.C. Number: 2.3.3.10

Salis rbs strength: 86314.57 au gacctcgggggggttcacATGACCATTGGCATCGACAAAATCAGCTTTTTTGTTCCGCCTTA CTATATCGACATGACCGCACTGGCCGAAGCACGTAATGTTGATCCGGGTAAATTTC ATATTGGTATTGGTCAGGATCAGATGGCCGTTAATCCGATTAGCCAGGATATTGTT ACCTTTGCAGCAAATGCAGCAGAAGCAATTCTGACCAAAGAAGATAAAGAAGCCAT CGATATGGTTATTGTTGGCACCGAAAGCAGCATTGATGAAAGCAAAGCAGCCGCA GTTGTTCTGCATCGTCTGATGGGTATTCAGCCGTTTGCACGTAGCTTTGAAATTAAA GAAGCATGTTACGGCGCAACCGCAGGTCTGCAGCTGGCAAAAAATCATGTTGCAC TGCATCCGGATAAAAAAGTTCTGGTTGTTGCAGCAGATATCGCCAAATATGGTCTG AATAGCGGTGGTGAACCGACCCAGGGTGCCGGTGCAGTTGCAATGCTGGTTGCAA GCGAACCGCGTATTCTGGCACTGAAAGAGGATAATGTTATGCTGACGCAGGATAT CTATGATTTTTGGCGTCCGACCGGTCATCCGTATCCGATGGTTGATGGTCCGCTGA GCAATGAAACCTATATTCAGAGCTTTGCACAGGTGTGGGATGAACATAAAAAACGT ACCGGTCTGGATTTCGCAGATTATGATGCACTGGCCTTTCATATTCCGTATACCAAA ATGGGTAAAAAAGCACTGCTGGCGAAAATTAGCGATCAGACCGAAGCCGAACAAG AACGTATCCTGGCACGTTATGAAGAAAGCATTATCTATAGCCGTCGTGTGGGTAAT CTGTATACCGGTAGCCTGTATCTGGGTCTGATTAGCCTGCTGGAAAATGCAACCAC CCTGACCGCTGGTAATCAGATTGGTCTGTTTAGCTATGGTAGCGGTGCCGTTGCA GAATTTTTCACAGGTGAACTGGTTGCAGGTTATCAGAATCATCTGCAGAAAGAAAC CCATCTGGCCCTGCTGGATAATCGTACCGAACTGAGCATTGCAGAATATGAAGCAA TGTTTGCAGAAACCCTGGATACCGATATTGATCAGACCCTGGAAGATGAACTGAAA TATAGCATTAGCGCCATTAATAACACCGTGCGTAGCTATCGTAATTAA

HMGS from Staphylococcus aureus (Tsuruta et al., 2009) E.C. Number: 2.3.3.10 Salis rbs strength: 92759.08 au 
ggatcttaaaggaggcaacaATGACCCTGGGCATTGATAAAATCAACTTCTATGTGCCGAAA TATTACGTGGATATGGCAAAACTGGCAGAAGCACGTCAGGTTGATCCGAACAAATT TCTGATTGGTATTGGTCAGACCGAAATGGCAGTTAGTCCGGTTAATCAGGATATTG TTAGCATGGGTGCAAATGCAGCCAAAGATATTATCACCGATGAAGATAAAAAAAAA ATCGGCATGGTTATCGTTGCAACCGAAAGCGCAGTTGATGCAGCAAAAGCAGCAG CAGTTCAGATTCATAATCTGCTGGGTATTCAGCCGTTTGCACGTTGTTTTGAAATGA AAGAAGCATGTTACGCAGCAACACCGGCAATTCAGCTGGCAAAAGATTATCTGGCA ACCCGTCCGAATGAAAAAGTTCTGGTTATTGCCACCGATACCGCACGTTATGGTCT GAATAGCGGTGGTGAACCGACCCAGGGTGCCGGTGCAGTTGCAATGGTTATTAGC CATAATCCGAGCATTCTGGCACTGAATGAAGATGCAGTTGCCTATACCGAAGATGT GTATGATTTTTGGCGTCCGACCGGTCATAAATATCCGCTGGTTGATGGTGCACTGA GCAAAGATGCATATATTCGTAGCTTTCAGCAGAGCTGGAATGAATATGCAAAACGT CAGGGTAAAAGCCTGGCAGATTTTGCAAGCCTGTGTTTTCATGTTCCGTTTACCAA AATGGGTAAAAAAGCCCTGGAAAGCATTATTGATAATGCCGATGAAACCACCCAAG AACGTCTGCGTAGCGGTTATGAGGATGCCGTTGATTATAACCGTTATGTGGGTAAC ATTTATACCGGTAGCCTGTATCTGAGCCTGATTAGCCTGCTGGAAAATCGTGATCT GCAGGCAGGCGAAACCATTGGTCTGTTTAGCTATGGTAGCGGTAGCGTTGGTGAG TTTTATAGCGCAACCCTGGTTGAAGGTTATAAAGATCATCTGGATCAGGCAGCACA TAAAGCACTGCTGAATAATCGTACCGAAGTTAGCGTTGATGCGTATGAAACCTTTTT CAAACGCTTCGATGATGTGGATTTTGATGAACAGCAGGATGCAGTTCATGAAGATC GCCATATCTTTTATCTGAGCAACATTGAAAACAATGTGCGCGAATATCATCGTCCG GAATAA

HMGR from Saccharomyces cerevisiae (Dueber et al., 2009) E.C. Number: 1.1.1.34 Salis rbs strength: 254196.2 au acagttaaggaggtaaaaATGGTGCTGACGAACAAAACCGTCATTAGCGGCAGCAAGGTG AAGTCTCTGAGCAGCGCCCAAAGCTCTAGCAGCGGCCCGTCTAGCAGCAGCGAG GAGGACGACAGCCGTGACATTGAGTCTCTGGACAAGAAGATCCGCCCGCTGGAG GAGTTAGAGGCCCTGCTGAGCAGCGGCAACACCAAGCAGCTGAAGAACAAGGAA GTTGCAGCGCTGGTGATCCACGGTAAGCTGCCACTGTATGCGCTGGAAAAGAAAC TGGGCGATACGACGCGTGCGGTCGCGGTGCGTCGCAAAGCCTTAAGCATCTTAG CGGAGGCCCCGGTGTTAGCCAGCGACCGCCTGCCGTACAAGAACTACGACTACG ACCGCGTGTTTGGCGCGTGCTGCGAGAATGTCATTGGCTACATGCCGTTACCGGT TGGTGTGATCGGCCCGCTGGTCATTGATGGCACGAGCTATCACATTCCAATGGCG ACCACGGAAGGTTGCTTAGTCGCCAGCGCCATGCGTGGCTGTAAGGCGATTAACG CCGGCGGTGGCGCGACGACCGTGTTAACCAAGGATGGTATGACGCGCGGTCCGG TCGTCCGCTTCCCAACGCTGAAGCGCAGCGGCGCGTGTAAGATTTGGCTGGATTC TGAGGAGGGCCAAAACGCGATCAAGAAAGCCTTCAACTCTACGAGCCGTTTCGCG CGTTTACAGCATATCCAGACCTGCCTGGCCGGCGACCTGCTGTTCATGCGCTTCC GCACCACCACGGGCGATGCGATGGGCATGAACATGATCAGCAAGGGCGTCGAAT ATAGCCTGAAACAAATGGTGGAAGAATATGGCTGGGAGGACATGGAGGTTGTCTC TGTGAGCGGCAACTATTGCACCGACAAGAAGCCGGCAGCCATTAACTGGATTGAG GGTCGCGGCAAAAGCGTCGTGGCAGAAGCGACCATCCCAGGCGACGTGGTCCGT AAGGTTCTGAAGAGCGACGTCAGCGCCCTGGTTGAGTTAAATATCGCGAAAAACC TGGTCGGCAGCGCGATGGCGGGCAGCGTGGGTGGCTTTAACGCACATGCAGCGA ATCTGGTTACGGCGGTTTTCTTAGCCTTAGGTCAGGACCCAGCCCAAAATGTCGAG 
AGCAGCAACTGCATTACCTTAATGAAAGAGGTTGACGGTGACCTGCGCATCAGCG TTTCTATGCCGTCTATCGAGGTCGGCACGATCGGCGGCGGCACCGTTTTAGAACC GCAAGGTGCGATGCTGGATCTGCTGGGCGTGCGCGGCCCACATGCAACGGCCCC AGGCACCAATGCCCGCCAACTGGCCCGTATCGTGGCCTGCGCGGTTCTGGCGGG TGAGCTGAGCCTGTGCGCCGCATTAGCCGCGGGCCATTTAGTTCAATCTCACATG ACCCACAACCGCAAGCCGGCAGAACCAACCAAGCCAAATAACCTGGACGCAACCG ACATTAACCGTCTGAAGGATGGCAGCGTCACGTGCATTAAAAGCGGCGCGCCATG A

HMGR from Staphylococcus aureus (Tsuruta et al., 2009) E.C. Number: 1.1.1.34 Salis rbs strength: 50296.95 au

cccgaaacaaggagggaaaaATGCAGAGCCTGGATAAAAACTTTCGTCATCTGAGCCGTC AGCAGAAACTGCAGCAGCTGGTTGATAAACAGTGGCTGAGCGAAGAACAGTTTAA CATTCTGCTGAATCATCCGCTGATTGATGAAGAAGTTGCAAACAGCCTGATTGAAA ATGTTATTGCACAGGGTGCACTGCCGGTTGGTCTGCTGCCGAACATTATTGTTGAT GATAAAGCATATGTGGTGCCGATGATGGTTGAAGAACCGAGCGTTGTTGCAGCAG CAAGCTATGGTGCAAAACTGGTTAATCAGACCGGTGGCTTTAAAACCGTTAGCAGC GAACGTATTATGATTGGCCAGATTGTTTTTGATGGTGTGGATGATACCGAAAAACT GAGCGCAGATATTAAAGCCCTGGAAAAACAAATTCATCAGATTGCCGATGAAGCCT ATCCGAGCATTAAAGCACGTGGTGGTGGTTATCAGCGTATTGCAATTGATACCTTT CCGGAACAGCAACTGCTGAGCCTGAAAGTGTTTGTTGATACCAAAGATGCAATGG GTGCCAATATGCTGAATACCATTCTGGAAGCAATTACCGCCTTTCTGAAAAATGAAT TTCCGCAGAGCGATATCCTGATGAGCATTCTGAGCAATCATGCAACCGCAAGCGTT GTTAAAGTTCAGGGTGAAATTGATGTTAAAGACCTGGCACGCGGTGAACGTACCG GTGAAGAGGTTGCCAAACGTATGGAACGTGCAAGCGTTCTGGCACAGGTTGATAT TCATCGTGCAGCAACCCATAATAAAGGTGTGATGAACGGTATTCATGCAGTTGTTC TGGCAACCGGTAATGATACCCGTGGTGCGGAAGCAAGCGCACATGCATATGCCAG CAAAGATGGTCAGTATCGTGGTATTGCAACCTGGCGTTATGATCAAGAACGTCAGC GTCTGATTGGTACAATTGAAGTTCCGATGACCCTGGCAATTGTTGGTGGTGGCACC AAAGTTCTGCCGATTGCAAAAGCAAGCCTGGAACTGCTGAATGTTGAAAGCGCACA AGAACTGGGTCATGTTGTTGCCGCAGTGGGTCTGGCCCAGAATTTTGCAGCATGT CGTGCACTGGTTAGCGAAGGTATCCAGCAGGGTCATATGAGTCTGCAGTATAAAA GCCTGGCCATTGTGGTTGGTGCCAAAGGTGATGAAATTGCGCAGGTTGCAGAAGC ACTGAAACAAGAACCGCGTGCAAATACCCAGGTTGCGGAACGTATTCTGCAGGAT CTGCGTAGCCAGCAGTAA

\begin{abstract}
HMGR from Pseudomonas mevalonii (Ma et al., 2011) E.C. Number: 1.1.1.88 Salis rbs strength: 52612.24 au agaattaaggaggtaataATGAGCTTGGACAGCCGCCTGCCAGCTTTTCGGAACCTGAGC CCCGCGGCGCGCCTGGATCATATTGGCCAGCTCTTGGGCCTGAGCCATGATGAC GTGAGCCTGCTGGCGAACGCGGGAGCGCTGCCGATGGATATTGCGAACGGCATG ATTGAGAACGTGATTGGCACCTTTGAACTGCCATACGCGGTGGCGAGCAACTTTCA GATTAATGGCCGGGACGTGCTGGTGCCGCTGGTGGTGGAGGAACCAAGCATTGT GGCGGCTGCTAGCTATATGGCGAAACTGGCGCGGGCGAACGGCGGCTTTACCAC CAGCAGCAGCGCGCCGCTGATGCACGCGCAGGTACAGATTGTGGGCATACAaGAT CCGTTGAATGCTCGCCTGAGCCTGCTGCGCCGCAAGGATGAGATTATAGAGCTGG
\end{abstract}


CGAACCGCAAAGATCAGCTCTTAAACAGCTTAGGCGGCGGCTGCCGCGATATTGA GGTGCATACCTTTGCGGACACCCCGCGGGGCCCGATGCTGGTGGCGCATCTGAT TGTGGACGTACGCGACGCGATGGGCGCGAACACCGTGAATACCATGGCGGAAGC GGTAGCGCCGCTGATGGAGGCGATTACCGGAGGCCAGGTACGCCTGCGCATACT GAGCAACCTGGCGGATCTGCGCCTGGCGAGGGCGCAGGTtCGGATAACACCGCA GCAACTGGAGACAGCGGAGTTTTCAGGCGAAGCTGTGATTGAGGGCATTTTGGAT GCGTATGCGTTTGCTGCGGTIGATCCCTATCGCGCGGCGACCCATAACAAAGGCAT TATGAATGGCATTGATCCCCTGATTGTGGCGACAGGCAACGATTGGCGGGCTGTG GAGGCGGGCGCGCACGCGTACGCGTGCCGCTCAGGACATTATGGCAGCCTGACC ACCTGGGAGAAAGATAACAACGGCCACCTTGTGGGCACCCTGGAGATGCCGATGC CAGTAGGCCTGGTGGGCGGCGCGACCAAGACCCACCCGCTGGCGCAACTGAGCC TGCGCATTTTAGGCGTGAAGACAGCGCAGGCGTTGGCTGAAATAGCGGTGGCGG TAGGCCTGGCGCAAAACTTAGGAGCGATGCGCGCGCTGGCGACCGAGGGCATTC AGCGCGGCCATATGGCGCTGCACGCGCGCAATATAGCGGTGGTGGCGGGCGCG AGGGGCGACGAAGTGGATTGGGTAGCGCGGCAGCTTGTGGAGTATCATGATGTG CGCGCGGATCGCGCGGTAGCTCTGCTGAAGCAAAAACGCGGCCAATAA

HMGR from Streptococcus pneumoniae (Yoon et al., 2009) E.C. Number: 1.1.1.88 Salis rbs strength: 75413.39 au gcacttaaggaggtatcgATGAAGATCAGCTGGAACGGTTTTAGCAAAAAAAGCTACCAGG AGCGGCTTGAGCTCTTGAAAGCGCAAGCTCTCTTAAGCCCAGAGCGGCAGGCGA GCCTTGAGAAGGATGAACAAATGAGCGTAACGGTAGCCGACCAACTTAGCGAGAA CGTCGTAGGTACGTTTAGCCTCCCATATTCACTTGTGCCaGAGGTCTTAGTCAATG GCCAAGAATACACTGTGCCtTATGTAACGGAAGAACCIAGCGTAGTGGCTGCTGCA TCATATGCATCAAAAATCATCAAGCGCGCCGGCGGCTTTACGGCCCAGGTCCATC AACGGCAAATGATTGGGCAAGTCGCATTGTATCAGGTGGCGAACCCAAAATTGGC TCAGGAGAAGATTGCATCAAAGAAAGCTGAGCTCTTGGAGTTTGCAAACCAGGCAT ATCCAAGCATCGTGAAACGCGGTGGCGGGGCTCGCGATCTTCATGTCGAGCAAAT CAAAGGGGAACCGGACTTTCTTGTGGTGTATATTCATGTCGATACTCAAGAAGCAA TGGGCGCAAACATGCTTAATACTATGCTTGAAGCATTAAAACCGGTCTTAGAAGAA CTCAGCCAAGGTCAAAGCCTTATCGGTATCCTCTCAAATTACGCTACTGATAGCCTT GTAACGGCCTCATGCCGGATGGCATTTCGGTACTTGTCACGGCAGAAGGATCAGG GTCGGGAGATTGCTGAGAAAATTGCTTTGGCGAGCCAATTTGCTCAAGCcGATCCA TACCGGGCGGCGACGCATAACAAAGGTATTTTTAACGGCATTGATGCTATTTTGAT TGCAACGGGCAACGACTGGCGCGCAATCGAAGCGGGGGCACATGCATTTGCAAG CCGGGATGGTCGGTATCAGGGCTTAAGCTGTTGGACACTCGACTTGGAACGGGAA GAATTGGTCGGCGAGATGACTCTTCCtATGCCAGTCGCTACGAAGGGCGGGAGCA TCGGGCTCAATCCGCGCGTCGCGTTCAGCCATGATTTGTTAGGTAACCCAAGCGC ACGGGAATTGGCACAAATTATCGTATCAATCGGCTTGGCACAGAACTTTGCCGCAC TTAAAGCGTTAGTCAGCACAGGaATCCAACAGGGGCACATGAAATTACAAGCAAAA TCATTAGCGCTTTTAGCGGGGGCGAGCGAAAGCGAAGTGGCGCCITTAGTCGAGC GGCTCATCAGCGATAAAACTTTTAATTTGGAGACGGCACAACGGTATCTCGAAAAC TTACGGAGCTAA

HMGR from Bordetella petrii (Ma et al., 2011) E.C. Number: 1.1.1.88 Salis rbs strength: 79502.36 au 
ggattaggaggaaggcgtATGAGCACCGATGCAAAAAATAGCCGTATTAGCGGCTTTCACA AAGATGATATTCCGACCCGTCTGGCACGTGTTGCAGCATTTGCAGGTCTGGATGAT GAAACCGTTCAGCATCTGGCAAATATGGGTAATCTGGACCCGCAGCTGGCAGATC GTCTGATTGAAAATGTTGTTGCAACCCTGAATGTGCCGATTGGTATTGCAACCAAT ATGAAAGTTGATGGCGAAGATGTTCTGGTTCCGATGGCAACCGAAGAAAGCAGCG TTGTTGCAGCCGTTTGTAATGCAGCACGTCAGTGTTATGATCAGGGTGGTTTTACC ACCAGTATGAGCGGTAGCCTGATGATTGCACAGGTTCAGCTGGTTGATGTTCCGG ATGCAGCACATGCACGTATGCGTATTCTGGAACATAAAGCCGAAGTTAAAGCACTG TGTGATGATTGTGATCCGCTGCTGGTTAAACTGGGTGGTGGTCTGCAGGATGTTGA AGTTCGTATTGTTGATGCAGCCGGTGGTCCGATGGTTGTTACCCATCTGATTGTTG ATACCCGTGATGCAATGGGTGCAAATGCAGTTAATAGCATGGCAGAAAAACTGGCA CCGCATATTGAAAGCTGGACCGGTGGTCGTGTTTATCTGCGCATTCTGAGCAATCT GGCCGATCGTCGCCTGGCACGCGCACGTGCAGTTTGGACCTGTGATGCCATTGGT GGTGCAAGCGTTCGTGATGGTATTATTAGCGCATATCGTTTTGCAGCAGCAGATCC GTATCGTGCAGCAACCCATAACAAAGGTATTATGAATGGTGTTAGCGCAGTTGTTC TGGCAACCGGTAATGATACACGTGCCGTTGAAGCCGGTGCACATGCATATGCCGC ACGTAAAGGTTGGTATAGCAGCCTGACCGATTGGGAAGTTACCGCAGAAGGTCAT CTGGCAGGCACCCTGGAAATGCCGATGGCAGTTGGTCTGGTGGGTGGTGCCACA AAACTGCATCCGACCGCACGTGCCTGTCTGAAAATTCTGGGTGTTAGCACCGCAG AACGGCTGGCACGCCTGATTGCAGCAGTGGGTCTGGCACAGAATTTTAGCGCACT GAAAGCACTGGCAACCACCGGTATTCAGAAAGGTCATATGAGCCTGCATGCACAG AATATTGCAATGATGGCAGGCGCAGTTGGTGATGAAATTGAACCGGTTGCAAAAGC CCTGGTTGCACAGGGTGCAGTTCGTGTTGATGTTGCAGAAGCAGAACTGGCACGT CTGCGTGGTCAGGGTTAA

\section{ACAT/HMGR bifunctional enzyme ( $m v a E$ gene) from Enterococcus faecalis} (Zurbriggen et al., 2012) E.C. Number: 2.3.1.9 /1.1.1.34

Salis rbs strength: 78530.65 au

gctcccaaggaggtaagcATGAAAACCGTTGTGATTATCGATGCCCTGCGTACGCCGATTG GTAAATACAAAGGCTCTCTGTCGCAGGTTAGCGCGGTCGATCTGGGCACGCACGT

TACCACCCAACTGCTGAAACGCCACTCGACAATTTCCGAGGAAATCGATCAAGTGA TTTTTGGCAATGTGCTTCAGGCGGGAAACGGCCAGAACCCGGCCCGTCAAATCGC CATCAACTCAGGACTCTCTCATGAGATCCCTGCTATGACGGTTAACGAAGTGTGTG GTAGTGGAATGAAGGCGGTGATCCTGGCGAAACAATTAATTCAGCTGGGCGAAGC GGAAGTCTTGATCGCTGGCGGCATCGAAAACATGAGCCAGGCACCtAAACTCCAG CGCTTCAATTACGAAACCGAAAGTTATGATGCGCCGTTCTCCAGCATGATGTACGA TGGTCTGACGGATGCTTTCAGCGGCCAGGCCATGGGCCTGACAGCAGAAAACGTC GCGGAAAAATATCATGTGACCCGTGAAGAGCAGGATCAGTTCAGTGTCCACAGCC AGCTGAAGGCGGCGCAGGCACAGGCGGAAGGCATTTTTGCCGATGAAATTGCAC CGTTAGAGGTGTCAGGTACGCTGGTCGAAAAAGATGAAGGGATTCGCCCTAACTC CTCCGTTGAAAAATTAGGCACGTTGAAAACCGTTTTTAAAGAAGATGGGACAGTGA CCGCCGGCAACGCGTCCACGATTAATGATGGGGCCTCAGCCCTGATCATTGCGTC TCAGGAGTACGCGGAGGCCCACGGTCTGCCATATCTGGCTATTATCCGTGACTCG GTGGAGGTGGGGATCGATCCGGCGTACATGGGCATTTCACCgATTAAGGCTATCC AAAAACTGCTCGCACGTAACCAATTAACGACTGAAGAGATTGATCTGTATGAAATC AATGAAGCATTTGCGGCAACCTCAATTGTTGTGCAACGTGAACTGGCCTTGCCaGA 
AGAAAAAGTGAACATCTATGGTGGTGGTATTTCACTGGGTCATGCGATCGGCGCTA CAGGCGCCCGCCTGTTAACTAGCTTGAGCTACCAATTAAACCAGAAAGAAAAAAAA TATGGAGTGGCCTCACTGTGCATCGGTGGTGGCTTGGGCCTGGCGATGCTGCTTG AACGTCCGCAGCAGAAGAAAAACTCTCGGTTTTACCAAATGAGCCCGGAGGAACG CCTCGCGTCGTTACTGAACGAGGGTCAGATTAGTGCCGATACCAAAAAAGAATTTG AAAATACTGCATTGAGCTCACAGATCGCGAATCATATGATCGAAAATCAAATTAGTG AAACCGAGGTTCCAATGGGCGTTGGTTTGCACCTTACGGTGGACGAGACGGACTA CTTAGTTCCGATGGCTACCGAAGAACCAAGCGTAATTGCAGCCTTAAGCAACGGA GCGAAAATCGCTCAGGGCTTCAAAACGGTCAACCAACAGCGCCTGATGCGGGGC CAGATCGTGTTTTACGATGTTGCCGATGCCGAATCACTGATTGATGAATTGCAAGT GCGCGAAACGGAAATTTTCCAACAAGCCGAACTCTCATACCCATCCATTGTAAAAC GCGGTGGCGGGTTACGTGACTTGCAATACCGCGCATTCGACGAATCATTCGTATC TGTTGATTTTCTGGTGGATGTAAAAGACGCAATGGGCGCAAACATTGTCAACGCGA TGCTTGAGGGTGTCGCGGAACTCTTTCGTGAATGGTTTGCCGAACAGAAAATTCTG TTTTCTATCCTTTCAAATTACGCCACCGAATCAGTGGTTACCATGAAAACCGCAATC CCGGTTTCACGTCTGAGTAAAGGTAGCAACGGTCGTGAAATTGCGGAAAAAATCGT GTTAGCCTCACGCTATGCCAGTCTCGACCCTTATCGCGCTGTAACCCATAACAAGG GTATTATGAACGGTATCGAAGCAGTCGTTCTGGCTACCGGCAATGATACCCGTGCA GTGTCTGCATCCTGTCACGCTTTTGCGGTTAAAGAAGGGCGTTACCAGGGCCTGA CAAGCTGGACTCTCGATGGAGAACAGTTAATCGGGGAAATCTCCGTGCCGTTGGC CCTGGCGACAGTTGGGGGCGCAACGAAGGTGCTGCCAAAGAGTCAAGCGGCAGC CGACTTATTAGCGGTTACGGATGCAAAAGAATTATCACGGGTCGTGGCCGCTGTG GGTCTGGCTCAGAACCTGGCTGCCTTACGCGCACTCGTTAGCGAGGGTATCCAGA AAGGCCATATGGCTCTGCAAGCGCGGTCCCTCGCTATGACCGTTGGGGCTACGG GCAAAGAGGTTGAAGCCGTAGCACAGCAACTGAAACGTCAGAAAACCATGAACCA AGACCGTGCGTTAGCGATCCTGAATGATTTACGTAAACAGTAA 
Appendix S2. Sequences of scars for Datsenko-Wanner knockouts

Uppercase - chromosomal DNA

Lowercase - scar sequence

Blue - PCR primers for Fig5B gel

Red - FRT sequence

IdhA $[1,417,413<-1,418,402]$

ATGAATTTTTCAATATCGCCATAGCTTTCAATTATATTTGAAATTTTGTAAAATATTTT

TAGTAGCTTAAATGTGATTCAACATCACTGGAGAAAGTCTTgtgtaggctggagctgcttcgaa gttcctatactttctagagaataggaacttcggaataggaactaaggaggatattcatatggaccatggctaattcccat TCTTGCCGCTCCCCTGCAACCCAGGGGAGCTGATTCAGATAATCCCCAATGACCTT TCATTCTCTATTCTTAAAATAGTCCTGAGTCAGAAACTGTAATT

acs $[4,192,825<-4,194,783]$

CCCCTATGTGTAACAAATAACCACACTGTGAATGTTGTCTTTAATCAATTGTAAGTG CATGTAAAATACCACTTTAGAGTTAGTTAGTATCTTCCTCTTTTTCAACAGCATGCAT AACTGCATGTTCCTCAAAGAATTAATCAACTTTTTGTTGCTGACCTTCAAAAATTACC CTGCCGTTTATTTGCACAATTCTACTTTTGCGTGATCTGTCGCCCAAATACTAAACA AAACTGCCAATACCCCTACATTTAACGCTTATGCCACATATTATTAACATCCTACAA GGAGAACAAAAGCgtgtaggctggagctgcttcgaagttcctatactttctagagaataggaacttcggaata ggaactaaggaggatattcatatggaccatggctaattcccatCCCACAATTGCCGGATGCGAGTCGG TAACGGTTTGTAGGCCTGATAAGACGCGACAGCGTCGCATCAGGCATTGATTGCC GGATGCGGCGTATAACGCCTTATCCGGCCTACATTCGGCAAGGGTTACCCGAGCG TTAACCTTCTCCCATAAGGGAGCGGGAATTAAAACAATCCCTACATTACCTCTGGA GAATCTGTGATGAATGGTACGATTTATCAGCGGATAGAAGACAATGCGCATTT

ackA-pta $[2,300,723$-> 2,304,144]

CAGGTATCCTTTAGCAGCCTGAAGGCCTAAGTAGTACATATTCATTGAGTCGTCAA ATTCATATACATTATGCCATTGGCTGAAAATTACGCAAAATGGCATAGACTCAAGAT ATTTCTTCCATCATGCAAAAAAAAATTTGCAGTGCATGATGTTAATCATAAATGTCG GTGTCATCATGCGCTACGCTCTATGGCTCCCTGACGTTTTTTTAAGCCACGTATCAAT TATAGGTACTTCCgtgtaggctggagctgcttcgaagttcctatactttctagagaataggaacttcgaactgca ggtcgacggatccccggaattaattctcatgtttgacagcttatcactgatcagtgaattaatggcgatgacgcatcctcacg ataatatccgggtaggcgcaatcactttcgtctctactccgttacaaagcgaggctgggtatttcccggcctttctgttatccga aatccactgaaagcacagcggctggctgaggagataaataataaacgaggggctgtatgcacaaagcatcttctgttga gttaagaacgagtatcgagatggcacatagccttgctcaaattggaatcaggtttgtgccaataccagtagaaacagacg aagaagctagctttgcactggattgcgaggctttgccatggctaattcccatTCTCGTCATCATCCGCAGCTT TGCGCTGCGGATATCTGAACCGGAAATAATCACTATTTCCGGTTTTTTATTCTCTTA ATCTGCATTAATCCTTTCTGATTATCTTGCTTAACTGCGCTGCATCAATGAATTGCG CCATTTCACTTTGCATACTTACCACTTTGTTTTGTGCAAGGGAATATTTGCGCTATG TCCGCAATCACTGAATCCAAACCAACAAGAAGATGGGCAATGCCCGATACGTTGGT GATTATCTTTTTTGTTGCCATTTTAACCAGTCTTGCCACCTGGGTAGTTCCG

Comment: the scar sequence for ackA-pta is longer than the expected 103bp sequence found in IdhA and acs. The additional scar DNA contains homology to ORF60 (unknown 
function, found in the pKD46 plasmid and BL21(DE3) genome). However, the ackA-pta reading frame is clearly absent and thus, no protein is expressed.

\section{Appendix S3. Calculation of theoretical yield}

\section{Maximum theoretical titer of mevalonate:}

$$
200 \mathrm{mM} \text { glucose } \cdot \frac{2 \mathrm{molmevalonate}}{3 \mathrm{~mol} \text { glucose }}+200 \mathrm{mM} \text { acetate } \cdot \frac{1 \text { mol mevalonate }}{3 \mathrm{~mol} \text { aceate }}=200 \mathrm{mM} \text { mevalonate }
$$

\section{Actual molar yield:}

$$
\frac{93.8 \mathrm{mM} \mathrm{mevalonate} \cdot \frac{6 \mathrm{~mol} \text { carbon }}{\mathrm{mol} \mathrm{mevalonate}}}{200 \mathrm{mM} \mathrm{glucose} \cdot \frac{6 \mathrm{~mol} \mathrm{carbon}}{\mathrm{mol} \text { glucose }}+200 \mathrm{mM} \text { acetate } \cdot \frac{2 \mathrm{~mol} \mathrm{carbon}}{\text { mol acetate }}}=0.352 \frac{\text { mol carbon product }}{\text { mol carbon substrate }}
$$

\section{Theoretical molar yield:}

$\frac{200 \mathrm{mM} \mathrm{mevalonate} \cdot \frac{6 \mathrm{~mol} \text { carbon }}{\mathrm{mol} \mathrm{mevalonate}}}{200 \mathrm{mM} \mathrm{glucose} \cdot \frac{6 \mathrm{~mol} \mathrm{carbon}}{\mathrm{mol} \text { glucose }}+200 \mathrm{mM} \text { acetate } \cdot \frac{2 \mathrm{~mol} \mathrm{carbon}}{\text { mol acetate }}}=0.750 \frac{\text { theoretical maximum mol carbon product }}{\text { mol carbon substrate }}$

\section{Theoretical yield (\%):}

$$
\frac{93.8 \mathrm{mM} \text { mevalonate }}{200 \mathrm{mM} \text { mevalonate }}=\frac{0.352 \mathrm{~mol} C \text { product per mol C susbrate }}{0.750 \mathrm{~mol} \mathrm{C} \mathrm{theo.} \mathrm{max.} \mathrm{product} \mathrm{per} \mathrm{mol} \mathrm{C} \mathrm{substrate}}=46.9 \% \text { theroical yield }
$$




\section{References:}

Calhoun, K. A., Swartz, J. R., 2005. Energizing cell-free protein synthesis with glucose metabolism. Biotechnology and bioengineering. 90, 606-613.

Datsenko, K. A., Wanner, B. L., 2000. One-step inactivation of chromosomal genes in Escherichia coli K-12 using PCR products. Proceedings of the National Academy of Sciences. 97, 6640-6645.

Dueber, J. E., Wu, G. C., Malmirchegini, G. R., Moon, T. S., Petzold, C. J., Ullal, A. V., Prather, K. L., Keasling, J. D., 2009. Synthetic protein scaffolds provide modular control over metabolic flux. Nature biotechnology. 27, 753-759.

Jewett, M. C., Calhoun, K. A., Voloshin, A., Wuu, J. J., Swartz, J. R., 2008. An integrated cell-free metabolic platform for protein production and synthetic biology. Molecular systems biology. 4.

Jewett, M. C., Swartz, J. R., 2004. Mimicking the Escherichia coli cytoplasmic environment activates long-lived and efficient cell-free protein synthesis. Biotechnology and bioengineering. 86, 19-26.

Kay, J. E., Jewett, M. C., 2015. Lysate of engineered Escherichia coli supports highlevel conversion of glucose to 2, 3-butanediol. Metabolic engineering. 32, 133142.

Ma, S. M., Garcia, D. E., Redding-Johanson, A. M., Friedland, G. D., Chan, R., Batth, T. S., Haliburton, J. R., Chivian, D., Keasling, J. D., Petzold, C. J., Lee, T. S., Chhabra, S. R., 2011. Optimization of a heterologous mevalonate pathway through the use of variant HMG-CoA reductases. Metabolic engineering. 13, 588597.

Martin, V. J., Pitera, D. J., Withers, S. T., Newman, J. D., Keasling, J. D., 2003. Engineering a mevalonate pathway in Escherichia coli for production of terpenoids. Nature biotechnology. 21, 796-802.

Paddon, C., Westfall, P., Pitera, D., Benjamin, K., Fisher, K., McPhee, D., Leavell, M., Tai, A., Main, A., Eng, D., 2013. High-level semi-synthetic production of the potent antimalarial artemisinin. Nature. 496, 528-532.

Rodriguez, S., Denby, C. M., Vu, T. v., Baidoo, E. E., Wang, G., Keasling, J. D., 2016. ATP citrate lyase mediated cytosolic acetyl-CoA biosynthesis increases mevalonate production in Saccharomyces cerevisiae. Microbial cell factories. 15, 1.

Tabata, K., Hashimoto, S.-I., 2004. Production of mevalonate by a metabolicallyengineered Escherichia coli. Biotechnology letters. 26, 1487-1491.

Tsuruta, H., Paddon, C. J., Eng, D., Lenihan, J. R., Horning, T., Anthony, L. C., Regentin, R., Keasling, J. D., Renninger, N. S., Newman, J. D., 2009. High-level production of amorpha-4, 11-diene, a precursor of the antimalarial agent artemisinin, in Escherichia coli. PloS one. 4, e4489.

Westfall, P. J., Pitera, D. J., Lenihan, J. R., Eng, D., Woolard, F. X., Regentin, R., Horning, T., Tsuruta, H., Melis, D. J., Owens, A., 2012. Production of amorphadiene in yeast, and its conversion to dihydroartemisinic acid, precursor to the antimalarial agent artemisinin. Proceedings of the National Academy of Sciences. 109, E111-E118. 
Woo, H. M., Murray, G. W., Batth, T. S., Prasad, N., Adams, P. D., Keasling, J. D., Petzold, C. J., Lee, T. S., 2013. Application of targeted proteomics and biological parts assembly in E. coli to optimize the biosynthesis of an anti-malarial drug precursor, amorpha-4, 11-diene. Chemical Engineering Science. 103, 21-28.

Xiong, M., Schneiderman, D. K., Bates, F. S., Hillmyer, M. A., Zhang, K., 2014. Scalable production of mechanically tunable block polymers from sugar. Proceedings of the National Academy of Sciences. 111, 8357-8362.

Yoon, S.-H., Lee, S.-H., Das, A., Ryu, H.-K., Jang, H.-J., Kim, J.-Y., Oh, D.-K., Keasling, J. D., Kim, S.-W., 2009. Combinatorial expression of bacterial whole mevalonate pathway for the production of $\beta$-carotene in E. coli. Journal of biotechnology. 140, 218-226.

Zurbriggen, A., Kirst, H., Melis, A., 2012. Isoprene production via the mevalonic acid pathway in Escherichia coli (Bacteria). BioEnergy Research. 5, 814-828. 